\title{
Photonic band-gap structures of core-shell simple cubic crystals from holographic lithography
}

\author{
Jun Hyuk Moon and Shu Yang ${ }^{\text {a) }}$ \\ Department of Materials Science and Engineering, University of Pennsylvania, 3231 Walnut Street, \\ Philadelphia, Pennsylvania 19104 \\ Seung-Man Yang \\ National Creative Research Initiative Center for Integrated Optofluidic Systems \\ and Department of Chemical and Biomolecular Engineering, Korea Advanced Institute of Science \\ and Technology, 305-701 Guseong-dong, Yuseong-gu, Daejeon, Korea
}

(Received 26 July 2005; accepted 19 January 2006; published online 20 March 2006)

\begin{abstract}
We report the investigation of photonic band-gap properties of a core-shell simple cubic structure (air core with a dielectric shell) using a two-parameter level-set approach. The proposed structure can be obtained by partially backfilling high refractive index materials into a polymeric template fabricated by multibeam interference lithography. We find that the shell formation in the inverted simple cubic structure increases the complete photonic band-gap width by $10 \%-20 \%$ in comparison to that of a completely filled structure. The band gap between the fifth and sixth bands begins to appear at a refractive index contrast of 2.7. This study suggests the importance to investigate the core-shell formation in three-dimensional photonic crystals through backfilling, which may offer an additional control over their photonic band-gap properties. (C) 2006 American Institute of Physics.
\end{abstract}

[DOI: $10.1063 / 1.2187438$ ]

A three-dimensional (3D) photonic crystal (PC) that possesses a complete photonic band gap (PBG) is highly desirable to confine, manipulate, and guide photons for a broad range of applications, including low-threshold lasers, ${ }^{1}$ lightemitting devices, ${ }^{2}$ optical biosensors, ${ }^{3}$ and microphotonic devices. ${ }^{4}$ To fabricate 3D photonic crystals, many methods have been used, such as self-assembly of colloidal particles and block copolymers, ${ }^{5,6}$ layer-by-layer photolithography, ${ }^{7}$ direct-write assembly, ${ }^{8}$ and two-photon lithography. ${ }^{9}$ Among them, interference (or holographic) lithography ${ }^{10}$ is a very promising candidate, which enables rapid production of defect-free 3D crystals over a large area with considerable control over both lattice size and symmetry.

In holographic lithography, the focused laser beams interfere to generate periodic patterns in photosensitive polymers, which, however, typically have low refractive indices. For example, the refractive index of SU- 8 photoresist is $\sim 1$.6. Therefore, backfilling with a higher index material is needed, followed by removal of the polymer template to fabricate PC with a complete PBG. Chemical vapor deposition and liquid phase deposition were used to infiltrate high index materials, such as titania $(n=2.5-3.0),{ }^{11}$ silicon $(n=3.5),{ }^{12}$ and germanium $(n=4.0)^{13}$ into sacrificial polymeric templates. It should be noted that the deposition reaction usually occurs between the liquid or vapor precursors and the corresponding functional groups on the template surface. A shell structure is formed at first, which grows continuously normal to the initial surface to fill the interstitial voids [e.g., Figs. 1(b) and 1(c)]. Previously it was found that in a partially filled inverted face-centered cubic opal, the shell formation could enhance the PBG, and the bandwidth of a directional $L$ stopgap could be tuned with the core-to-shell ratio. ${ }^{14,15}$

In this letter, we use a two-parameter level-set approach to investigate the $\mathrm{PBG}$ properties of core-shell formation in a

${ }^{a)}$ Electronic mail: shuyang@ @eas.upenn.edu simple cubic $P(P m 3 m)$ structure. Of the various PC structures, diamond $D$ and gyroid $G$ have received much attention due to their large gap width and robustness. ${ }^{16-19}$ However, it is not straightforward to produce these structures by multibeam interference lithography, because each term in the level-set equation is dependent on all 3D $(x, y$, and $z)$ coordinates. The accessible lattice size is limited by the choice of beam parameters and the wavelength of the laser. In comparison, the simple cubic $P(P m 3 m)$ structure can be size scalable via triple exposures of a two-beam interference pattern, where the angle between the beams of the individual gratings can be varied. ${ }^{20}$ This primitive structure shows a relatively wide and full PBG with a maximum gap to midgap ratio of $13 \%$ between the fifth and sixth bands for a dielectric contrast of 13:1 and a volume fraction of $0.26 .{ }^{21}$ Moreover, the pseudogaps along the (100) direction ( $X$ gap) in the simple cubic structure are very wide and appeared over a large range of filling ratios. ${ }^{22}$ The position of the pseudogap between the second and third bands is more sensitive to the lattice constant than that of the $L$ gap in the fcc structure, which makes the simple cubic structure attractive for tunable photonic crystals and optical sensors. In this letter we investigate the photonic band-gap properties of the core-shell $P$ structure.
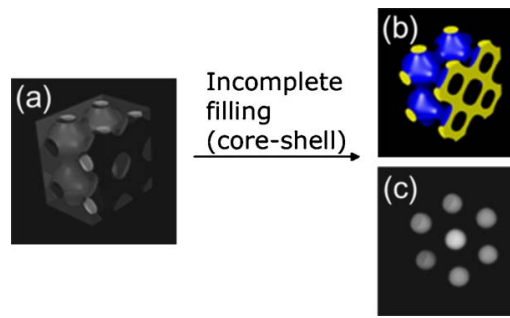

Complete filling



FIG. 1. (Color online) Templating method to produce PC of high refractive index materials. (a) Template, (b) air core/dielectric shell, (c) air core, and (d) completely filled structure. 
The formation of the $P$ structure was analyzed via the level-set approach. ${ }^{23}$ In this approach, the surface of a porous dielectric structure is represented by the solution to scalarvalued functions $F(x, y, z)$ of three independent variables, and the volume fraction can be controlled by varying the parameter, $t$. Therefore, the structure is defined as

$$
F(x, y, z)>t \text { for dielectric and } F(x, y, z)<t \text { for air. }
$$

Using a similar approach, we propose two level-set surfaces of interference patterns to simulate triply periodic structures with a shell-like morphology given by

$$
t_{1}<F(x, y, z)<t_{2} \text { for dielectric, }
$$

and

$$
t_{1}>F(x, y, z) \text { and } t_{2}<F(x, y, z) \text { for air. }
$$

This structure is divided into the inner-core (air) and the outer-shell (dielectric) surfaces defined by $t_{2}$ and $t_{1}$, respectively. The resulting two level surfaces share the same normal vectors such that they are in parallel to each other. Because the shell morphology can be formed from either liquid phase deposition or chemical vapor deposition (CVD) process, the use of two $t$ parameters closely approximates the deposited surfaces. For example, Fig. 1 shows a schematic of a simple cubic structure formed from interference lithography, and the evolution of its replicas from air-core-shell to completely filled structures. Because the sum of a level surface and the surface translated by half a lattice period gives a constant maximum value in the level-set equation, i.e., $F(-x,-y,-z)=-F(x, y, z)$, the volume fraction is symmetrically related to the exposure intensity. Therefore, the desired volume fraction of the primitive photonic crystals with high refractive index [Fig. 1(c)] can be obtained from templates [Fig. 1(a)] with controlled exposure intensity.

In the band-gap calculation, we use the level-set surface of $F(x, y, z)=\sin (x)+\sin (y)+\sin (z)$ for Eq. (2) and two parameters, $t_{1}$ and $t_{2}$, for the outer and inner surfaces of dielectric materials $\left(n=n_{d}\right)$, respectively. ${ }^{20}$ The band structures of the PC are calculated using the MIT photonic bands (MPB) software package. ${ }^{24}$ We first calculate the complete PBG with only a single parameter $t_{1}$ (i.e., completely filled structure) ranging from 0.6 to 0.9. A band-gap appears between the fifth and sixth bands. The maximum PBG is found at $t_{1}$ $=0.83-0.85$ with $n_{d}=3.50$, and the corresponding volume fraction is $0.25-0.26$, which agrees well with the results from literature. ${ }^{20}$ Then, we optimize $t_{2}$ to maximize the complete band-gap width while keeping the $t_{1}$ fixed (i.e., core-shell structure). For $t_{1}$ ranging from 0.65 to 0.90 , the maximum PBG is obtained at $t_{2}=2.5-2.7$, which is smaller than $t_{2}$ $=3.0$ required for a completely filled simple cubic structure [see Fig. 2(a)]. Therefore, the formation of shell in a simple cubic structure increases the complete PBG by $10 \%-20 \%$ within the range of aforementioned $t_{1}$. By analogy, it was reported that the band gap of two-dimensional(2D) square lattice can be increased by the introduction of an additional square lattice of smaller unit atoms, which effectively reduces the crystal symmetry. ${ }^{25}$ Figure 2 (b) shows the calculated photonic band structure of a core-shell $P$ structure with the maximum PBG. It is found that the introduction of an air core lifts the sixth band especially at wave vector $M$ (the bottom of the sixth band), resulting in the increase of PBG.

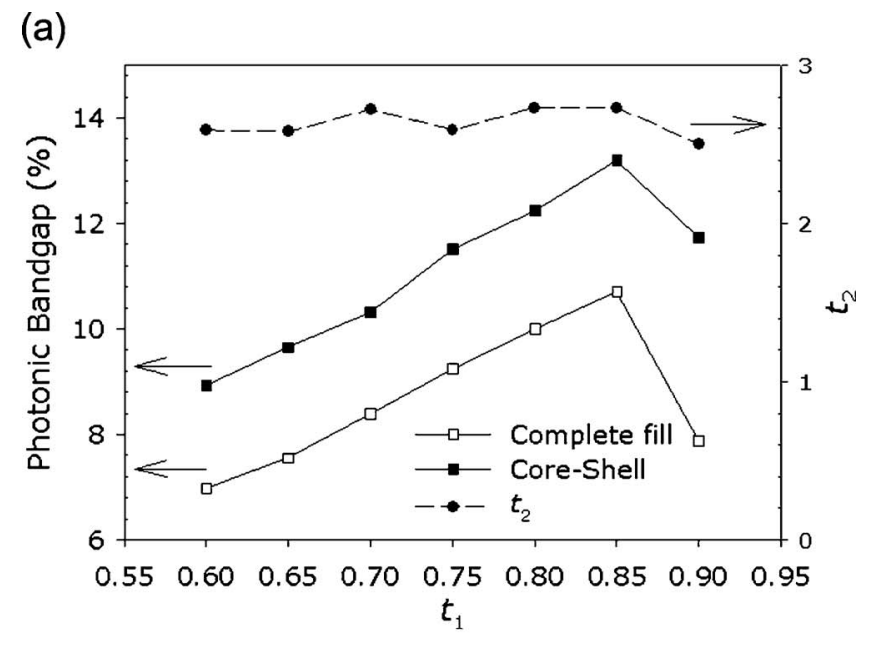

(b)

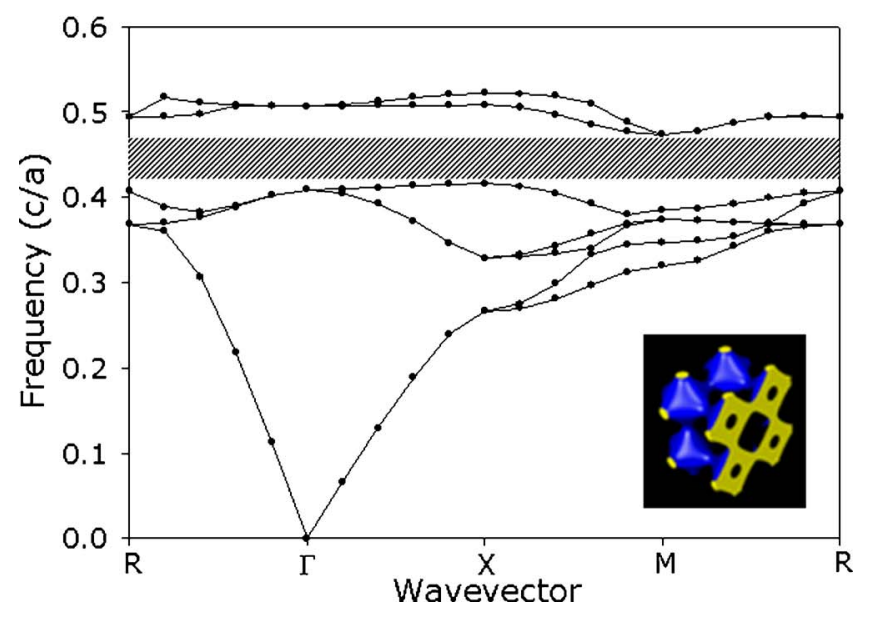

FIG. 2. (Color online) (a) Complete photonic band gap of the simple cubic lattice with core-shell and completely filled structures, respectively. The $t_{2}$ is optimized with respect to $t_{1}$ to maximize the band-gap width. (b) Photonic band structure of the simple cubic lattice with $t_{1}=0.85$ and $t_{2}=2.7$ or $25 \%$ filled, and $n_{d}=3.50$, showing complete PBG between the fifth and sixth bands.

The inset shows the optimized structure. Compared to the volume fraction of 0.26 for completely filled structures, the optimized core-shell $P$ structure has a slightly lower volume fraction of 0.25 . However, such photonic band-gap enhancement is not observed in core-shell diamond and gyroid structures.

Figure 3 shows the gap/midgap ratios of the $P$ structure shown in Fig. 2(b) as a function of the refractive index. The band-gap is calculated from the $P$ surface with the largest PBG, that is $t_{1}=0.84$ for complete filling, and $t_{1}=0.84$ and $t_{2}=2.70$ for a core-shell structure. Previously, it was reported that the minimum refractive index required to open a complete PBG is approximately 2.0 for $D, 2.2$ for $G$, and 2.8 for $P$ structures. $^{21,22}$ In the core-shell $P$ structure, the minimum index contrast required to open a complete PBG is found to be less than 2.7. Thus, in practice, a simple cubic photonic crystal with a complete PBG at optical wavelengths can be achieved by the deposition of anatase titania into photoresist templates. ${ }^{11}$

In conclusion, we investigated the photonic band-gap properties of a core-shell simple cubic structure using a twoparameter level-set approach. The 3D structure is defined by license or copyright; see http://apl.aip.org/about/rights_and_permissions 


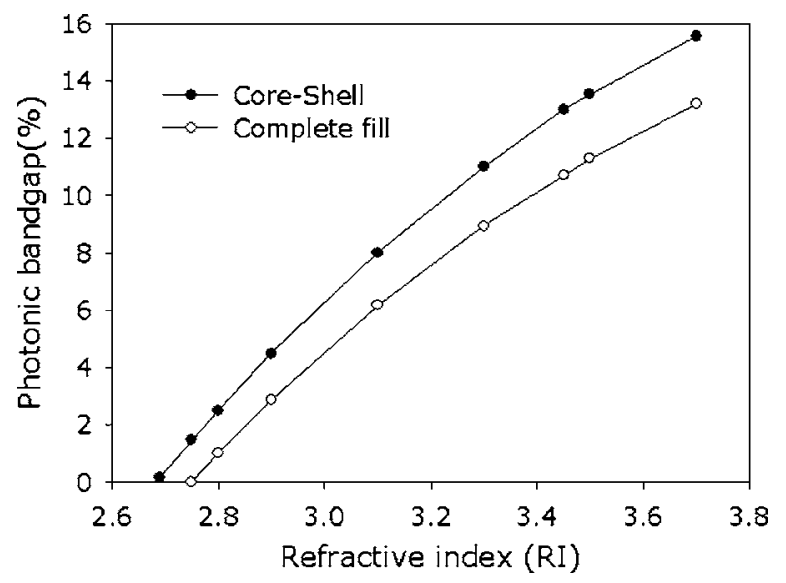

FIG. 3. Gap/midgap ratio as a function of refractive index contrast in the simple cubic structure shown in Fig. 2(b).

the inner-core (air) and the outer-shell (dielectric) surfaces. The proposed structure can be fabricated by backfilling a sacrificial polymer template created by multibeam interference lithography. The photonic band-gap width is found increased in the core-shell primitive structure in comparison to that of the completely filled one. A complete band gap between the fifth and sixth bands begins to appear at a refractive index contrast of 2.7. This suggests that the core-shell formation may offer additional controls over photonic properties. The two-parameter level-set approach presented in this letter may provide a useful guidance for the fabrication of $3 \mathrm{D}$ photonic structures through backfilling.

This work is supported by the Office of Naval Research (ONR) through the MURI program, Grant No. N00014-050303, and the Korea Research Foundation postdoc fellowship (J.H.M.), Grant No. KRF-2005-000-10299. S.M.Y. acknowledges supports from National R\&D Project of Nano Science and Technology of Korea, BK-21 Program, CUPSERC and Creative Research Initiative Program of the Ministry of Science \& Technology. The authors thank Martin Maldovan and Chaitanya K. Ullal (MIT) for useful discussions.
${ }^{1}$ H. G. Park, S. H. Kim, S. H. Kwon, Y. G. Ju, J. K. Yang, J. H. Baek, S. B. Kim, and Y. H. Lee, Science 305, 1444 (2004).

${ }^{2}$ B. J. Matterson, J. M. Lupton, A. F. Safonov, M. G. Salt, W. L. Barnes, and I. D. W. Samuel, Adv. Mater. (Weinheim, Ger.) 13, 123 (2001).

${ }^{3}$ Y. J. Lee and P. V. Braun, Adv. Mater. (Weinheim, Ger.) 15, 563 (2003).

${ }^{4}$ M. Francois, J. Danglot, B. Grimbert, P. Mounaix, M. Muller, O. Vanbesien, and D. Lippens, Microelectron. Eng. 61-62, 537 (2002).

${ }^{5}$ V. L. Colvin, MRS Bull. 26, 637 (2001).

${ }^{6}$ A. C. Edrington, A. M. Urbas, P. DeRege, C. X. Chen, T. M. Swager, N. Hadjichristidis, M. Xenidou, L. J. Fetters, J. D. Joannopoulos, Y. Fink, and E. L. Thomas, Adv. Mater. (Weinheim, Ger.) 13, 421 (2001).

${ }^{7}$ K. Aoki, H. T. Miyazaki, H. Hirayama, K. Inoshita, T. Baba, K. Sakoda, N. Shinya, and Y. Aoyagi, Nat. Mater. 2, 117 (2003).

${ }^{8}$ G. M. Gratson, M. J. Xu, and J. A. Lewis, Nature (London) 428, 386 (2004).

${ }^{9}$ B. H. Cumpston, S. P. Ananthavel, S. Barlow, D. L. Dyer, J. E. Ehrlich, L. L. Erskine, A. A. Heikal, S. M. Kuebler, I. Y. S. Lee, D. McCordMaughon, J. Q. Qin, H. Rockel, M. Rumi, X. L. Wu, S. R. Marder, and J. W. Perry, Nature (London) 398, 51 (1999).

${ }^{10}$ M. Campbell, D. N. Sharp, M. T. Harrison, R. G. Denning, and A. J. Turberfield, Nature (London) 404, 53 (2000); J. H. Moon and S. Yang, J. Macromol. Sci., Part C: Polym. Rev. 45, 351 (2005).

${ }^{11}$ J. Wijnhoven, L. Bechger, and W. L. Vos, Chem. Mater. 13, 4486 (2001).

${ }^{12}$ A. Blanco, E. Chomski, S. Grabtchak, M. Ibisate, S. John, S. W. Leonard,

C. Lopez, F. Meseguer, H. Miguez, J. P. Mondia, G. A. Ozin, O. Toader, and H. M. van Driel, Nature (London) 405, 437 (2000).

${ }^{13}$ H. Miguez, F. Meseguer, C. Lopez, M. Holgado, G. Andreasen, A. Mifsud, and V. Fornes, Langmuir 16, 4405 (2000).

${ }^{14}$ K. Busch and S. John, Phys. Rev. E 58, 3896 (1998).

${ }^{15}$ K. P. Velikov, A. Moroz, and A. van Blaaderen, Appl. Phys. Lett. 80, 49 (2002).

${ }^{16}$ M. Maldovan and E. L. Thomas, Nat. Mater. 3, 593 (2004).

${ }^{17}$ M. Maldovan, C. K. Ullal, W. C. Carter, and E. L. Thomas, Nat. Mater. 2, 664 (2003).

${ }^{18}$ L. Martin-Moreno, F. J. Garcia-Vidal, and A. M. Somoza, Phys. Rev. Lett. 83, 73 (1999).

${ }^{19}$ D. N. Sharp, A. J. Turberfield, and R. G. Denning, Phys. Rev. B 68, 205102 (2003).

${ }^{20}$ C. K. Ullal, M. Maldovan, E. L. Thomas, G. Chen, Y. J. Han, and S. Yang, Appl. Phys. Lett. 84, 5434 (2004).

${ }^{21}$ M. Maldovan, A. M. Urbas, N. Yufa, W. C. Carter, and E. L. Thomas, Phys. Rev. B 65, 165123 (2002).

${ }^{22}$ R. Biswas, M. M. Sigalas, K. M. Ho, and S. Y. Lin, Phys. Rev. B 65, 205121 (2002).

${ }^{23}$ C. K. Ullal, M. Maldovan, M. Wohlgemuth, and E. L. Thomas, J. Opt. Soc. Am. A 20, 948 (2003).

${ }^{24} \mathrm{http} / / /$ ab-initio.mit.edu/mpb/.

${ }^{25}$ C. M. Anderson and K. P. Giapis, Phys. Rev. Lett. 77, 2949 (1996). 